Communications in Physics, Vol. 26, No. 2 (2016), pp. 111-120

DOI:10.15625/0868-3166/26/2/8461

\title{
A MODEL OF BULK SUPERSYMMETRY IN WARPED SPACETIME
}

\author{
TRAN MINH HIEU ${ }^{\dagger}$ \\ Hanoi University of Science and Technology, No. 1 Dai Co Viet road, Hanoi, Vietnam \\ ${ }^{\dagger}$ E-mail: hieu.tranminh@hust.edu.vn
}

Received 04 July 2016

Accepted for publication 12 August 2016

\begin{abstract}
The Randall-Sundrum solution to the hierarchy problem in the brane-world scenario has made a significant impact on the development of model building. We investigate in this paper a model of supersymmetry in a 5D spacetime with the Randal-Sundrum warped metric. The minimal supersymmetric extension of the standard model (MSSM) superfields propagate in the bulk between the UV and IR branes at separated orbifold fixed points, while the hidden sector in charge of supersymmetry breaking is confined on the UV brane. We derive the $4 D$ effective action from the original 5D action of the underlying theory. The effective 4D Yukawa couplings and all the soft supersymmetry breaking terms are calculated in terms of the 5D theory's coefficients. We comment on the important role of the bulk mass parameter in the relation with the geometrical properties of particles.
\end{abstract}

Keywords: supersymmetry, Randall-Sundrum, warped spacetime.

Classification numbers: 11.30.Pb, 12.60.-i.

\section{INTRODUCTION}

Although the standard model (SM) is very successful in describing the interactions between elementary particles, it is obviously not the ultimate theory of the nature. There are many theoretical and experimental problems that the SM cannot solve by itself. They are motivations for physics beyond the SM. One of the important questions needed to be addressed relates to the stability of the electroweak scale. It is well known that a theory with a scalar field such as the SM suffers from quadratic divergences when taking into account quantum corrections. According to that, the correction of the scalar mass which represents the electroweak scale is proportional to a much higher scale of some underlying theory like the grand unification scale or the Planck scale, leading to the instability of the electroweak scale. This is the so-called gauge hierarchy problem or the problem of naturalness.

In an attempt to solve this problem, Randall and Sundrum (RS) proposed a brane-world scenario with a warped metric as a solution to the Einstein-Hilbert action [1]. The electroweak 
scale can be naturally generated from a much higher mass scale of the underlying 5D theory due to the background geometry. The original RS model puts all the SM fields in an infrared (IR) brane located at one of the orbifold fixed points, only gravitational field can propagate in the bulk. Other possibilities were investigated after that where the SM fields live in the bulk while the SM Higgs boson is confined on the brane [2]. In these works [3, 4], the authors considered the supersymmetric (SUSY) extension of the RS model in the formalisms of component field and superfield respectively. The SUSY RS models solve the gauge hierarchy problem by the SUSY, while the RS background plays the role to lower down the cutoff scale. Therefore, the warping effect does not need to be as large as in the original RS model. Moreover, the mass spectrum and couplings can be determined from the geometrical configurations of fields, providing a possibility to have more universality in the underlying 5D theory, and bringing physics and geometry closer together. Assuming R-parity conservation, the SUSY framework predicts the lightest weakly interacting sparticle to be a dark matter candidate which was not taken into account in the original RS model. These models provide a flexible framework and interesting predictions for the phenomenological study [5]. A deep connection between these models and the string theory was also pointed out [6].

In this paper, we examine a setup of the minimal SUSY extension of the SM (MSSM) in the RS spacetime. All the MSSM superfields live in the entire bulk of the 5D spacetime. For breaking the SUSY, a hidden sector is put on the ultra-violet (UV) brane. The SUSY breaking source is communicated to the MSSM sector via the gravity mediation by introducing various contact couplings suppressed by the Planck scale. From the underlying 5D action, we derive the effective 4D action. Subsequently, we calculate the effective 4D Yukawa couplings and other soft SUSY breaking terms. The $\mu$-term problem is avoided in this model thanks to the Giudice-Masiero mechanism [7].

Structure of this paper is as follows. In Sec. II, we present the model setup with bulk superfields in the Randall-Sundrum background and introduce the contact couplings between the hidden and the MSSM sector which is in charge of the SUSY breaking mediation. In Sec. III, we derive the 4D effective action and soft SUSY breaking terms from the 5D particle profiles and couplings. We comment on the role of bulk mass parameters in determining the localization of fields in the extra dimension and the 4D effective couplings in Sec. IV. Lastly, Sec. V is devoted to conclusion.

\section{MODEL SETUP}

We consider a 5-dimensional spacetime $\left(x^{\mu}, y\right)$. The $y$ coordinate is periodic and takes values as angles along a circle with a radius $R$ such that $-\pi \leq y \leq \pi$. By the action of $Z_{2}$ parity, $y$ is identified with $-y$. This is the $S_{1} / Z_{2}$ orbifold where the fifth dimension is compactified on. This orbifold has two fixed points at $y=0$ and $y=\pi$. Supposing there are two $(3+1)$ dimensional branes ${ }^{1}$ located at these orbifold fixed points, their effects on the bulk gravitational metric are important and must be taken into account. A solution to the Einstein's equation of this braneworld configuration is given by a non-factorizable metric [1]:

$$
d s^{2}=e^{-2 R \sigma} \eta_{\mu \nu} d x^{\mu} d x^{v}-R^{2} d y^{2},
$$

\footnotetext{
${ }^{1}$ They are often referred to as "3-branes"
} 
where $\sigma=k|y|$, and $1 / k$ is the curvature radius. This metric reveals a property that the spacetime is indeed a slide of 5-dimensional Anti-de Sitter (AdS) space. It relates any 5D mass scales $M_{5}$ to the corresponding 4D mass scale $M_{4}$ via the warp factor $e^{-k R \pi}$. Especially, the relation between the 5D Planck scale and the 4D Planck scale is given as

$$
M_{P 4}^{2}=\frac{M_{P 5}^{3}}{k}\left(1-e^{-2 k R \pi}\right) .
$$

Within this context, the induced metrics at two boundaries result in the distant effective scales associated with each brane. The effective cutoff on the UV brane at $y=0$ boundary is the 5D Plank scale $M_{P 5}$, while that for the IR brane at $y=\pi$ boundary is $M_{P 5} e^{-k R \pi}$. In other words, the hierarchy between those effective scales is due to the small overlap of the graviton wave function in the 5th dimension (namely, the warp factor) with the IR brane compared to that with the UV brane.

Here we assume that all the MSSM superfields live in the entire bulk of the AdS space, while the SUSY breaking sector only lives on the UV brane. Since the gravitino's wave function localizes more toward the UV brane, the placement of the SUSY breaking sector results in heavy gravitinos. Hence the theory can avoid the gravitino-LSP (lightest sparticle) scenario, and the dark matter is the lightest neutralino which can be in thermal equilibrium. For the sake of simplicity, we suppose that the existence of bulk fields does not modify the AdS background metric above.

A 5D supermultiplet is a function of $\left(x^{\mu}, y, \theta, \bar{\theta}\right)$. Each 5D $N=1$ supermultiplet can be written in terms of familiar "4D-like" $N=1$ supermultiplets, each of them is expressed with the dependence on $y$ as a label. The Kaluza-Klein (KK) decomposition for a certain 5D supermuliplet tells us how their $y$-dependences look like. In this expansion, each 5D field corresponds to an infinite tower of $4 \mathrm{D}$ fields with increasing masses. Only the zero modes of the $Z_{2}$-even 5D fields are massless. They are used to describe the MSSM fields when all symmetries are manifest.

In the 5D theory's point of view, supermultiplets can be classified into gauge (vector) supermultiplets or hypermultiplets. 5D gauge supermultiplets are used to describe gauge bosons and gauginos. Each one of this type contains a 4D-like vector supermultiplet:

$$
V(x, y, \theta)=-\theta \sigma^{\mu} \bar{\theta} A_{\mu}(x, y)-i \bar{\theta}^{2} \theta \lambda_{1}(x, y)+i \theta^{2} \bar{\theta} \bar{\lambda}_{1}(x, y)+\frac{1}{2} \bar{\theta}^{2} \theta^{2} D(x, y),
$$

and a 4D-like chiral supermultiplet:

$$
\chi(x, y, \theta)=\frac{1}{\sqrt{2}}\left(\Sigma(x, y)+i A_{5}(x, y)\right)+\sqrt{2} \theta \lambda_{2}(x, y)+\theta^{2} F_{\chi}(x, y) .
$$

They transform as an adjoint representation of some gauge group. Under the action of $Z_{2}$ parity, $V$ is even while $\chi$ is odd. Therefore, only the fields belonging to a vector supermultiplet have massless modes in the KK decomposition. The gauge invariant 5D action for gauge supermultiplet is as follows

$$
\begin{aligned}
S_{5}^{\text {gauge }}= & \int d^{5} x\left\{\frac{1}{4 g_{5}^{2}} \int d^{2} \theta R W^{\alpha} W_{\alpha}+\right.\text { h.c. } \\
& \left.+\frac{2}{g_{5}^{2}} \int d^{4} \theta \frac{e^{-2 R \sigma}}{2 R} \operatorname{Tr}\left[\left\{e^{V / 2}, \partial_{5} e^{-V / 2}\right\}+\frac{1}{\sqrt{2}}\left(e^{V / 2} \chi^{\dagger} e^{-V / 2}+e^{-V / 2} \chi e^{V / 2}\right)\right]^{2}\right\},
\end{aligned}
$$


where the $5 \mathrm{D}$ gauge coupling $g_{5}$ relates to the $4 \mathrm{D}$ gauge coupling $g_{4}$ by $g_{5}=\sqrt{2 \pi R} g_{4}$. From dimensional analysis, we note that $V(x, y, \theta)$ and $\chi(x, y, \theta)$ are dimensionless, while $g_{5}$ has dimen$\operatorname{sion}-\frac{1}{2}$.

The Higgs boson, matter fields, and their superpartners are described by 5D hypermultiplets. It includes a pair of 4D-like vector-like chiral superfields $\Phi$ and $\Phi^{c}$ :

$$
\begin{aligned}
\Phi(x, y, \theta) & =\phi(x, y)+\sqrt{2} \theta \psi(x, y)+\theta^{2} F_{\Phi}(x, y), \\
\Phi^{c}(x, y, \theta) & =\phi^{c}(x, y)+\sqrt{2} \theta \psi^{c}(x, y)+\theta^{2} F_{\Phi^{c}}(x, y) .
\end{aligned}
$$

We assume that they are $Z_{2}$-even and $Z_{2}$-odd respectively. Therefore, only the fields belonging to $\Phi$ have massless states as the zero modes in the KK decomposition. The 5D action for hypermultiplets and their gauge interaction is given by

$$
\begin{aligned}
S_{5}^{\text {matter }}= & \int d^{5} x\left\{\int d^{4} \theta R e^{-2 R \sigma}\left(\Phi^{\dagger} e^{-V} \Phi+\Phi^{c} e^{V} \Phi^{c^{\dagger}}\right)\right. \\
& \left.+\int d^{2} \theta e^{-3 R \sigma} \Phi^{c}\left[\partial_{5}-\frac{1}{\sqrt{2}} \chi-\left(\frac{3}{2}-c_{\Phi}\right) R \sigma^{\prime}\right] \Phi+\text { h.c. }\right\},
\end{aligned}
$$

where $c_{\Phi}$ is hypermultiplet bulk mass. By solving the SUSY vacuum condition, we obtain the y-dependence of the massless zero mode of a hypermultiplet:

$$
\Phi(x, y, \theta)=\varphi_{0}(x, \theta) e^{\left(\frac{3}{2}-c_{\Phi}\right) R \sigma}+\ldots
$$

In this equation, $\varphi_{0}$ has dimension $\frac{3}{2}$ as the dimension of an usual 5D hypermultiplet.

In order to preserve $N=15 \mathrm{D}$ SUSY in the bulk, the Yukawa interactions must be confined to the branes ${ }^{2}$. The action describing the Yukawa interactions between bulk hypermultiplets in 5D theory can be written as

$$
S_{5}^{\text {Yukawa }}=\int d^{5} x \int d^{2} \theta e^{-3 R \sigma} \frac{y_{123}}{M_{P 5}^{3 / 2}}[\delta(y)+\lambda \delta(y-\pi)] \Phi_{1} \Phi_{2} \Phi_{3} .
$$

The appearance of $M_{P 5}^{3 / 2}$ in the denominator is for the dimensional matching. To avoid the $\mu$ problem associated with the SUSY Higgs mass $\mu$ in the MSSM superpotential, we use the GiudiceMasiero mechanism [7] by imposing an $U(1)_{R}$-symmetry for the theory. According to that, the R-charges are assigned to $\theta$ and the MSSM hypermultiplets as in Table 1 . The R-symmetry forbids the existence of the term $\mu H_{u}^{h} H_{d}^{h}$ in the SUSY Lagrangian. This term only emerges after the SUSY is broken in the hidden sector, and the value of $\mu$ will be determined by the SUSY breaking source.

Table 1. R-charges of $\theta$ and MSSM hypermultiplets.

\begin{tabular}{|c|c|c|c|c|c|c|c|c|}
\hline$\theta$ & $Q_{i}^{h}$ & $U_{i}^{h}$ & $D_{i}^{h}$ & $L_{i}^{h}$ & $E_{i}^{h}$ & $H_{u}^{h}$ & $H_{d}^{h}$ & $X$ \\
\hline 1 & 1 & 1 & 1 & 1 & 1 & 0 & 0 & 0 \\
\hline
\end{tabular}

On the UV brane, the superfield $X$ of the hidden sector is assigned the R-charge 0 . Therefore the R-charge of its F-term, $F_{X}$, is -2 . Via some hidden dynamics, this F-term acquires nonzero

\footnotetext{
${ }^{2} N=15 \mathrm{D}$ SUSY is equivalent to $N=24 \mathrm{D}$ SUSY. Due to the orbifold, it is reduced to $N=14 \mathrm{D}$ SUSY on
} the branes. 
vacuum expectation value (VEV), $\left\langle F_{X}\right\rangle$. The emergence of such a nonzero constant $\left\langle F_{X}\right\rangle$ explicitly breaks SUSY as well as R-symmetry. After that, the soft SUSY breaking terms of the MSSM will be switched on via various contact interactions between $X$ on the boundary and the bulk MSSM supermultiplets. It is worth to notice that the original contact terms must be R-symmetric when SUSY is manifest. There are various ways to write the contact terms in the action between the visible and hidden sectors, however we only consider the possibilities generating the usual MSSM soft SUSY breaking terms.

In general, the action that describes the interaction between gauge supermultiplets and the hidden field $X$ is of the form:

$$
S_{5}^{X g}=\int d^{5} x\left[\int d^{2} \theta d_{a} \frac{X}{M_{P 5}} W^{a \alpha} W_{\alpha}^{a}+\text { h.c. }\right] \delta(y) .
$$

The contact terms between Higgs hypermultiplets and $X$ field are written as follows

$$
\begin{aligned}
S_{5}^{X h}=\int d^{5} x \int d^{4} \theta & \left\{\left[d_{\mu} \frac{X^{\dagger}}{M_{P 5}^{2}} H_{u}^{h} H_{d}^{h}+d_{B_{\mu}} \frac{X^{\dagger} X}{M_{P 5}^{3}} H_{u}^{h} H_{d}^{h}+\text { h.c. }\right]\right. \\
& +\left[d_{A}^{H_{u}} \frac{X+X^{\dagger}}{M_{P 5}^{2}} H_{u}^{h^{\dagger}} H_{u}^{h}+d_{m}^{H_{u}} \frac{X^{\dagger} X}{M_{P 5}^{3}} H_{u}^{h \dagger} H_{u}^{h}\right. \\
& \left.\left.+d_{A}^{H_{d}} \frac{X+X^{\dagger}}{M_{P 5}^{2}} H_{d}^{h \dagger} H_{d}^{h}+d_{m}^{H_{d}} \frac{X^{\dagger} X}{M_{P 5}^{3}} H_{d}^{h^{\dagger}} H_{d}^{h}\right]\right\} \delta(y),
\end{aligned}
$$

and those between matter hypermultiplets and the hidden sector:

$$
S_{5}^{X m}=\int d^{5} x \int d^{4} \theta\left[\left(d_{A}^{\Phi}\right)_{i j} \frac{X+X^{\dagger}}{M_{P 5}^{2}} \Phi_{i}^{\dagger} \Phi_{j}+\left(d_{m}^{\Phi}\right)_{i j} \frac{X^{\dagger} X}{M_{P 5}^{3}} \Phi_{i}^{\dagger} \Phi_{j}\right] \delta(y),
$$

where $\Phi$ stands for $\left\{Q^{h}, U^{h}, D^{h}, L^{h}, E^{h}\right\}$ hypermultiplets, and $\{i, j\}$ are generation indices. The below contact terms correspond to 4-point couplings between the ordinary hypermultiplets and the hidden sector field:

$$
\begin{aligned}
S_{5}^{X a}=\int d^{5} x \int d^{2} \theta\{ & \frac{\left(a_{u}\right)_{i j}}{M_{P 5}^{5 / 2}} X H_{u}^{h} Q_{i}^{h} U_{j}^{h}+\frac{\left(a_{d}\right)_{i j}}{M_{P 5}^{5 / 2}} X H_{d}^{h} Q_{i}^{h} D_{j}^{h} \\
& \left.+\frac{\left(a_{e}\right)_{i j}}{M_{P 5}^{5 / 2}} X H_{d}^{h} L_{i}^{h} E_{j}^{h}+\text { h.c. }\right\} \delta(y) .
\end{aligned}
$$

\section{4D EFFECTIVE THEORY}

The effective $4 \mathrm{D}$ action is obtained from the $5 \mathrm{D}$ action by integrating over the extra 5 th dimension $y$. The massless zero modes of the 4D-like supermultiplets result in the usual 4D MSSM supermultiplets. In order to have a 4D effective theory with canonical kinetic terms, we redefine all zero-modes of the bulk hypermultiplets as follows

$$
\varphi_{0} \longrightarrow \sqrt{\frac{\left(1-2 c_{\Phi}\right) k}{2\left(e^{\left(1-2 c_{\Phi}\right) R k \pi}-1\right)}} \varphi_{0}=\mathscr{C}_{\Phi} \varphi_{0}
$$


where $\varphi_{0}$ represents the usual 4D MSSM fields: $H_{u}, H_{d}, Q_{i}, U_{i}, D_{i}, L_{i}, E_{i}$, and

$$
\mathscr{C}_{\Phi}=\sqrt{\frac{\left(1-2 c_{\Phi}\right) k}{2\left(e^{\left(1-2 c_{\Phi}\right) R k \pi}-1\right)}} .
$$

We note that the dimension of $\mathscr{C}_{\Phi}$ is $\frac{1}{2}$, therefore the redefinition changes the dimension of $\varphi_{0}$ to 1 as the dimension of an usual 4D chiral superfield. From now on, we only consider the massless zero-mode states which have interesting phenomenology at low energy, since all higher modes will be integrated out in the 4D effective theory.

After the $y$-integration and the above redefinition (15), the 4D effective action corresponding to the 5D Yukawa interactions (10) reads

$$
S_{4}^{\text {Yukawa }}=\int d^{4} x \int d^{2} \theta \varphi_{01} \varphi_{02} \varphi_{03} \frac{y_{123}}{M_{5}^{3 / 2}}\left[1+\lambda e^{\left(\frac{3}{2}-c_{\Phi 1}-c_{\Phi 2}-c_{\Phi 3}\right) k R \pi}\right] \mathscr{C}_{\Phi 1} \mathscr{C}_{\Phi 2} \mathscr{C}_{\Phi 3},
$$

where the combination $\left\{y_{123}, \varphi_{01}, \varphi_{02}, \varphi_{03}, c_{\Phi 1}, c_{\Phi 2}, c_{\Phi 3}\right\}$ stands for $\left\{\left(y_{u}\right)_{i j}, H_{u}, Q_{i}, U_{j}, c_{H u}, c_{Q i}, c_{U j}\right\}$, $\left\{\left(y_{d}\right)_{i j}, H_{d}, Q_{i}, D_{j}, c_{H d}, c_{Q i}, c_{D j}\right\}$, and $\left\{\left(y_{e}\right)_{i j}, H_{d}, L_{i}, E_{j}, c_{H d}, c_{L i}, c_{E j}\right\}$ of the MSSM. The 4D effective Yukawa couplings are now written in terms of 5D Yukawa couplings and bulk masses:

$$
\begin{aligned}
\left(Y_{u}\right)_{i j} & =\frac{\left(y_{u}\right)_{i j}}{M_{5}^{3 / 2}}\left[1+\lambda e^{\left(\frac{3}{2}-c_{H u}-c_{Q i}-c_{U j}\right) k R \pi}\right] \mathscr{C}_{H_{u}} \mathscr{C}_{Q_{i}} \mathscr{C}_{U_{j}}, \\
\left(Y_{d}\right)_{i j} & =\frac{\left(y_{d}\right)_{i j}}{M_{5}^{3 / 2}}\left[1+\lambda e^{\left(\frac{3}{2}-c_{H d}-c_{Q i}-c_{D j}\right) k R \pi}\right] \mathscr{C}_{H_{d}} \mathscr{C}_{Q_{i}} \mathscr{C}_{D_{j}}, \\
\left(Y_{e}\right)_{i j} & =\frac{\left(y_{e}\right)_{i j}}{M_{5}^{3 / 2}}\left[1+\lambda e^{\left(\frac{3}{2}-c_{H d}-c_{L i}-c_{E j}\right) k R \pi}\right] \mathscr{C}_{H_{d}} \mathscr{C}_{L_{i}} \mathscr{C}_{E_{j}}
\end{aligned}
$$

In a similar way, the $4 \mathrm{D}$ effective actions that generate the MSSM soft SUSY breaking terms are derived from Eqs. (11), (12), (13), and (14) respectively as follows

$$
\begin{gathered}
S_{4}^{X g}=\int d^{4} x\left[\int d^{2} \theta d_{a} \frac{X}{M_{P 5}} W^{a \alpha} W_{\alpha}^{a}+\text { h.c. }\right], \\
S_{4}^{X h}=\int d^{4} x \int d^{4} \theta\left\{\left[d_{\mu} \frac{X^{\dagger}}{M_{P 5}^{2}} H_{u} H_{d}+d_{B_{\mu}} \frac{X^{\dagger} X}{M_{P 5}^{3}} H_{u} H_{d}+\text { h.c. }\right] \mathscr{C}_{H_{u}} \mathscr{C}_{H_{d}}\right. \\
+\left[d_{A}^{H_{u}} \frac{X+X^{\dagger}}{M_{P 5}^{2}} H_{u}^{\dagger} H_{u}+d_{m}^{H_{u}} \frac{X^{\dagger} X}{M_{P 5}^{3}} H_{u}^{\dagger} H_{u}\right] \mathscr{C}_{H_{u}}^{2} \\
\left.+\left[d_{A}^{H_{d}} \frac{X+X^{\dagger}}{M_{P 5}^{2}} H_{d}^{\dagger} H_{d}+d_{m}^{H_{d}} \frac{X^{\dagger} X}{M_{P 5}^{3}} H_{d}^{\dagger} H_{d}\right] \mathscr{C}_{H_{d}}^{2}\right\}, \\
S_{4}^{X m}=\int d^{4} x \int d^{4} \theta\left[\left(d_{A}^{\Phi}\right)_{i j} \frac{X+X^{\dagger}}{M_{P 5}^{2}} \varphi_{0 i}^{\dagger} \varphi_{0 j}+\left(d_{m}^{\Phi}\right)_{i j} \frac{X^{\dagger} X}{M_{P 5}^{3}} \varphi_{0 i}^{\dagger} \varphi_{0 j}\right] \mathscr{C}_{\Phi_{i}} \mathscr{C}_{\Phi_{j}},
\end{gathered}
$$


where $\varphi_{0}$ stands for $\{Q, U, D, L, E\} 4 \mathrm{D}$ supermultiplets, and $\{i, j\}$ are generation indices. The triliear couplings generating terms read

$$
\begin{aligned}
S_{4}^{X a}=\int d^{4} x \int d^{2} \theta\{ & \frac{\left(a_{u}\right)_{i j}}{M_{P 5}^{5 / 2}} X H_{u} Q_{i} U_{j} \mathscr{C}_{H_{u}} \mathscr{C}_{Q_{i}} \mathscr{C}_{U_{j}}+\frac{\left(a_{d}\right)_{i j}}{M_{P 5}^{5 / 2}} X H_{d} Q_{i} D_{j} \mathscr{C}_{H_{d}} \mathscr{C}_{Q_{i}} \mathscr{C}_{D_{j}} \\
& \left.+\frac{\left(a_{e}\right)_{i j}}{M_{P 5}^{5 / 2}} X H_{d} L_{i} E_{j} \mathscr{C}_{H_{d}} \mathscr{C}_{L_{i}} \mathscr{C}_{E_{j}}+\text { h.c. }\right\} .
\end{aligned}
$$

After the $X$ field gets its VEV by some hidden mechanism, $\langle X\rangle=\theta^{2}\left\langle F_{X}\right\rangle$, the familiar MSSM soft SUSY breaking terms emerge from the contact terms of the $4 \mathrm{D}$ effective action. The gaugino masses are derived from Eq. (21) as

$$
M_{a}=-d_{a} g_{a}^{2} \frac{\left\langle F_{X}\right\rangle}{M_{P 5}}, \quad(a=1,2,3)
$$

Equation (23) leads to the scalar soft masses of sparticles:

$$
\left(m_{\Phi}\right)_{i j}^{2}=-\left(d_{m}^{\Phi}\right)_{i j} \frac{\left|\left\langle F_{X}\right\rangle\right|^{2}}{M_{P 5}^{3}} \mathscr{C}_{\Phi_{i}} \mathscr{C}_{\Phi_{j}}+\sum_{n=1}^{3} \frac{\left|\left\langle F_{X}\right\rangle\right|^{2}}{M_{P 5}^{4}}\left(d_{A}^{\Phi}\right)_{i n}\left(d_{A}^{\Phi}\right)_{n j} \mathscr{C}_{\Phi_{n}}^{2} \mathscr{C}_{\Phi_{i}} \mathscr{C}_{\Phi_{j}},
$$

where $\Phi$ stands for $Q, U, D, L, E$, and $i, j=\{1,2,3\}$. The soft masses of the Higgs sector can be obtained from (22) as

$$
\begin{aligned}
m_{H_{u}}^{2} & =-d_{m}^{H_{u}} \frac{\left|\left\langle F_{X}\right\rangle\right|^{2}}{M_{P 5}^{3}} \mathscr{C}_{H_{u}}^{2}+\frac{\left|\left\langle F_{X}\right\rangle\right|^{2}}{M_{P 5}^{4}}\left(d_{A}^{H_{u}}\right)^{2} \mathscr{C}_{H_{u}}^{4}, \\
m_{H_{d}}^{2} & =-d_{m}^{H_{d}} \frac{\left|\left\langle F_{X}\right\rangle\right|^{2}}{M_{P 5}^{3}} \mathscr{C}_{H_{d}}^{2}+\frac{\left|\left\langle F_{X}\right\rangle\right|^{2}}{M_{P 5}^{4}}\left(d_{A}^{H_{d}}\right)^{2} \mathscr{C}_{H_{d}}^{4}, \\
B_{\mu} & =d_{B_{\mu}} \frac{\left|\left\langle F_{X}\right\rangle\right|^{2}}{M_{P 5}^{3}} \mathscr{C}_{H_{u}} \mathscr{C}_{H_{d}}-d_{A}^{H_{u}} \frac{\left|\left\langle F_{X}\right\rangle\right|}{M_{P 5}^{2}} \mu \mathscr{C}_{H_{u}}^{2}-d_{A}^{H_{d}} \frac{\left|\left\langle F_{X}\right\rangle\right|}{M_{P 5}^{2}} \mu \mathscr{C}_{H_{d}}^{2}
\end{aligned}
$$

The ordinary SUSY Higgs mass originates from the SUSY breaking source $\left\langle F_{X}\right\rangle$ in the first term of Eq. (22) just as other soft SUSY breaking terms,

$$
\mu=d_{\mu} \frac{\left\langle F_{X}\right\rangle^{\dagger}}{M_{P 5}^{2}} \mathscr{C}_{H_{u}} \mathscr{C}_{H_{d}}
$$


The trilinear couplings A-terms arise from Eq. (24):

$$
\begin{aligned}
\left(A_{u}\right)_{i j}= & \frac{1}{\left(Y_{u}\right)_{i j}}\left\{\left(a_{u}\right)_{i j} \frac{\left\langle F_{X}\right\rangle}{M_{P 5}^{5 / 2}} \mathscr{C}_{H_{u}} \mathscr{C}_{Q_{i}} \mathscr{C}_{Q_{j}}\right. \\
& \left.-\left[d_{A}^{H_{u}}\left(Y_{u}\right)_{i j} \mathscr{C}_{H_{u}}^{2}+\sum_{n=1}^{3}\left(d_{A}^{Q}\right)_{n i}\left(Y_{u}\right)_{n j} \mathscr{C}_{Q_{n}} \mathscr{C}_{Q_{i}}+\sum_{n=1}^{3}\left(d_{A}^{U}\right)_{n j}\left(Y_{u}\right)_{i n} \mathscr{C}_{U_{n}} \mathscr{C}_{U_{j}}\right] \frac{\left\langle F_{X}\right\rangle}{M_{P 5}^{2}}\right\} \\
\left(A_{d}\right)_{i j}= & \frac{1}{\left(Y_{d}\right)_{i j}}\left\{\left(a_{d}\right)_{i j} \frac{\left\langle F_{X}\right\rangle}{M_{P 5}^{5 / 2}} \mathscr{C}_{H_{d}} \mathscr{C}_{Q_{i}} \mathscr{C}_{D_{j}}\right. \\
& \left.-\left[d_{A}^{H_{d}}\left(Y_{d}\right)_{i j} \mathscr{C}_{H_{d}}^{2}+\sum_{n=1}^{3}\left(d_{A}^{Q}\right)_{n i}\left(Y_{d}\right)_{n j} \mathscr{C}_{Q_{n}} \mathscr{C}_{Q_{i}}+\sum_{n=1}^{3}\left(d_{A}^{D}\right)_{n j}\left(Y_{d}\right)_{i n} \mathscr{C}_{D_{n}} \mathscr{C}_{D_{j}}\right] \frac{\left\langle F_{X}\right\rangle}{M_{P 5}^{2}}\right\} \\
\left(A_{e}\right)_{i j}= & \frac{1}{\left(Y_{e}\right)_{i j}}\left\{\left(a_{d}\right)_{i j} \frac{\left\langle F_{X}\right\rangle}{M_{P 5}^{5 / 2}} \mathscr{C}_{H_{d}} \mathscr{C}_{L_{i}} \mathscr{C}_{E_{j}}\right. \\
& \left.-\left[d_{A}^{H_{d}}\left(Y_{e}\right)_{i j} \mathscr{C}_{H_{d}}^{2}+\sum_{n=1}^{3}\left(d_{A}^{L}\right)_{n i}\left(Y_{e}\right)_{n j} \mathscr{C}_{L_{n}} \mathscr{C}_{L_{i}}+\sum_{n=1}^{3}\left(d_{A}^{E}\right)_{n j}\left(Y_{e}\right)_{i n} \mathscr{C}_{E_{n}} \mathscr{C}_{E_{j}}\right] \frac{\left\langle F_{X}\right\rangle}{M_{P 5}^{2}}\right\}
\end{aligned}
$$

We can see that all the soft SUSY breaking masses and couplings are scaled with the $F$-component's $\operatorname{VEV}\left\langle F_{X}\right\rangle$ of the hidden sector field.

\section{BULK MASS PARAMETERS}

In order to have 5D canonical Kahler potention in Eq. (8), we redefine the hypermultiplet as $\Phi \rightarrow \Phi e^{-R \sigma}$. Therefore, the massless zero mode wavefunction in the 5D spacetime is

$$
\left.\Phi(x, y, \theta)\right|_{\text {zero-mode }}=\varphi_{0}(x, \theta) e^{\left(\frac{1}{2}-c_{\Phi}\right) R \sigma} .
$$

The geometrical profile of the massless zero mode belonging to a hypermultiplet can be extracted from Eq. (34):

$$
f_{0}(y)=e^{\left(\frac{1}{2}-c_{\Phi}\right) k R y} .
$$

Given a background metric, it tells us that the wave function of particles in the extra dimension is uniquely determined by the bulk mass parameter $c_{\Phi}$. In Fig. 1, we demonstrate the geometrical profile for the case $k R=1$. We find that for the case $c_{\Phi}<\frac{1}{2}$, the zero mode superfield localizes more toward the IR brane at $y=\pi$. For the case $c_{\Phi}=\frac{1}{2}$, it has a flat configuration. And for the case $c_{\Phi}>\frac{1}{2}$, it localizes more toward the UV brane at $y=0$.

The bulk mass parameters determine not only the geometrical profiles of matter super-field, but also the effective 4D Yukawa couplings, $\mu$ and all the soft SUSY breaking terms via the geometrical coefficient $\mathscr{C}_{\Phi}$ as can be seen from Eqs. (18)-(20) and (25)-(33). We show in Fig. 2 the geometrical coefficient $\mathscr{C}_{\Phi}$ as a function of the bulk mass parameter $c_{\Phi}$ : 


$$
\mathscr{C}_{\Phi}=\sqrt{\frac{\left(1-2 c_{\Phi}\right) k}{2\left(e^{\left(1-2 c_{\Phi}\right) k R \pi}-1\right)}} .
$$

Here, we demonstrate different cases with the value $k R=1,2$ and 5 . We observe that $\mathscr{C}_{\Phi}$ is exponentially suppressed when $c_{\Phi} \rightarrow-\infty$, and increases when $c_{\Phi} \rightarrow+\infty$.

From these analyses, the bulk mass parameters can be considered as a bridge connecting the geometrical properties of particle's wave function in the extra dimension and their effective couplings and masses. This is an interesting feature of the model that will be useful for phenomenological study.

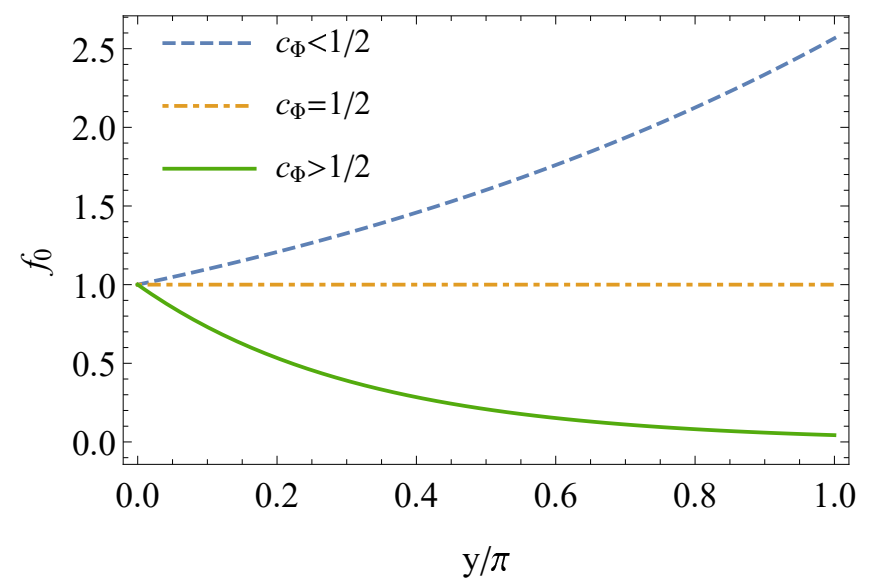

Fig. 1. The geometrical profile $f_{0}(y)$ for different possibilities of the bulk mass parameter $c_{\Phi}$. The demonstration is in the case $k R=1$.

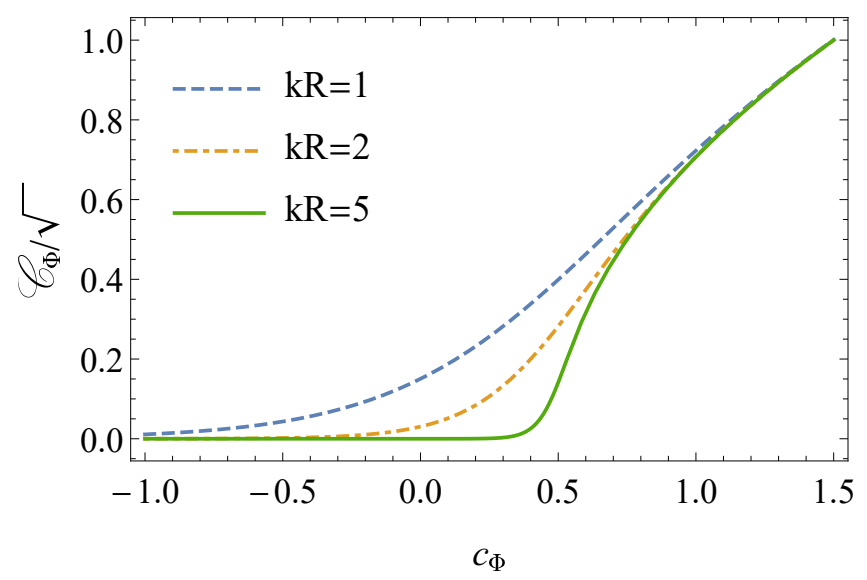

Fig. 2. The geometrical coefficient as a function of the bulk mass parameter. The function is undetermined at $c_{\Phi}=\frac{1}{2}$, however we can replace that point with the function's well-defined limit $c_{\Phi} \rightarrow \frac{1}{2}$. 


\section{CONCLUSION}

The RS braneworld scenario is a powerful approach to build a natural theory where distant scales are connected by the warp factor. In this paper, we have presented a model of bulk SUSY in the 5D AdS space with the RS warped metric. Gauge supermulitplets and hypermultiplets can propagate in the bulk, while the SUSY breaking sector is confined on the UV brane at one of the orbifold fixed points. From the 5D action of the model, we have derived the 4D effective action to describe the MSSM as a low energy sector constituted of massless zero modes in the KK decomposition. We have calculated all the soft SUSY breaking terms as well as $\mu$-term as results of the 5D contact interactions between the $X$ field and the bulk superfields. The relations between those terms, the geometrical profile of the MSSM superfields and their bulk mass parameters have opened a promising potential for phenomenological study.

\section{ACKNOWLEDGMENT}

The author would like to thank Prof. Nobuchika Okada for useful discussions. This work is supported in part by Vietnam National Foundation for Science and Technology Development (NAFOSTED) under the grant No. 103.01-2014.22.

\section{REFERENCES}

[1] L. Randall and R. Sundrum, Phys. Rev. Lett. 83 (1999) 3370 [hep-ph/9905221].

[2] W. D. Goldberger and M. B. Wise, Phys. Rev. D 60 (1999) 107505 [hep-ph/9907218]; S. Chang, J. Hisano, H. Nakano, N. Okada and M. Yamaguchi, Phys. Rev. D 62 (2000) 084025 [hep-ph/9912498].

[3] T. Gherghetta and A. Pomarol, Nucl. Phys. B 586 (2000) 141 [hep-ph/0003129]; T. Gherghetta and A. Pomarol, Nucl. Phys. B $6 \mathbf{0 2}$ (2001) 3 [hep-ph/0012378].

[4] N. Arkani-Hamed, T. Gregoire and J. G. Wacker, JHEP 0203 (2002) 055 [hep-th/0101233]; D. Marti and A. Pomarol, Phys. Rev. D 64 (2001) 105025 [hep-th/0106256].

[5] See for example: N. Okada and T. Yamada, Phys. Rev. D 84 (2011) 035005 [arXiv:1105.0241 [hep-ph]], and references therein.

[6] H. L. Verlinde, Nucl. Phys. B 580 (2000) 264 [hep-th/9906182]; M. J. Duff, J. T. Liu and K. S. Stelle, J. Math. Phys. 42 (2001) 3027 [hep-th/0007120]; M. Cvetic, M. J. Duff, J. T. Liu, H. Lu, C. N. Pope and K. S. Stelle, Nucl. Phys. B 605 (2001) 141 [hep-th/0011167].

[7] G. F. Giudice and A. Masiero, Phys. Lett. B 206 (1988) 480. 